\title{
Prevalence of musculoskeletal pain among competitive high school male water polo players in Kwa Zulu Natal, South Africa
}

\author{
Terry Jeremy Ellapen, Carl Stow, Natalie Macrae, Jessica Milne, Hendrick \\ Johan Van Heerden \\ University of Kwa-Zulu Natal, Faculty of Health Science, School of Physiotherapy, \\ Optometry and Sports Science
}

\section{Summary}

Introduction: Water polo is an aggressive, competitive aquatic game played by two teams of seven players with the intention to defeat the opposing team by scoring more goals which occasionally generate musculoskeletal pain and injury. Water polo incorporates swimming, treading, throwing the ball, in a volatile, dynamic aquatic domain which lends itself to a high degree of aggression and direct physical contact among opposing player.

This study documented the prevalence of water polo related musculoskeletal pain among competitive adolescent male water polo players residing in Kwa-Zulu Natal, South Africa $(\mathrm{n}=100)$.

Material and methods: Subjects completed a self-report musculoskeletal pain questionnaire, which gathered their demographical, epidemiological and exercise history over the last 12 months. The following descriptive statistics (mode, mean, frequency, percentages) and inferential statistics (chisquare set at a probability of 0.05 ) were employed to analyse the data.

Results: Seventy-two of the cohort sustained musculoskeletal pain within the last 12 months $(\mathrm{p}<0.001)$. The most prevalent anatomical sites that sustained water polo related musculoskeletal pain were; shoulder $(51.04 \%)$, knees $(23.95 \%)$ and vertebral column $(17.71 \%)(\mathrm{p}<0.0001)$. The predisposing mechanisms of producing this water polo related musculoskeletal pain were over-training $(88.00 \%)$, rapid rotational movements $(8.00 \%)$, collision with players $(2.66 \%)$, and being struck with the ball $(1.33 \%)(\mathrm{p}<0.001)$.

Conclusions: Competitive adolescent male water polo players residing in Kwa-Zulu Natal, South Africa experienced a high prevalence of water polo related shoulder, knee and vertebrae musculoskeletal pain.

Keywords:

water polo, swimming, musculoskeletal pain 


\section{Introduction}

Water polo is an aggressive, competitive aquatic game played by two teams of seven players with the intention to defeat the opposing team by scoring more goals. The players strategically pass the ball to gain an advantageous position which will allow them to the shoot ball beyond the reach of the opposing goal keeper into the goal net [1]. Water polo players predominately employ a modified free style swim stroke to move in the water. The players' arms and head are elevated above the water whilst the ball is positioned immediately in front of the player as they swim forward. This elevated swimming posture involves excessive shoulder abduction, elevation and internal rotation which place a tremendous load on the shoulder joint and surrounding tissue [2,3]. Players' spend a high percentage of the game treading water, employing a breast stroke kick to keep afloat. Water polo incorporates swimming, treading, throwing the ball in a volatile, dynamic aquatic domain which lends itself to a high degree of aggression and direct physical contact among opposing players [1].

The predisposing mechanisms of musculoskeletal pain and injury among water polo players are; over-use, direct physical trauma from collision with opposing players and being struck with ball $[4,5,6]$. Webster et al. [7] reports that the upper limbs are physically taxed more than the lower limbs in water polo, thereby increasing risk of musculoskeletal pain and/or injury. Mountjoy et al. [8] reported an incidence rate of 0.09 musculoskeletal pain and injuries per 1000 water polo players who participated in the 2009 FINA World Championships. Webster et al. [7] and Colville et al. [9] reported a musculoskeletal shoulder pain and injury prevalence ranging from $38 \%$ to $80 \%$. Clinicians have reported that the repetitive nature of the swimming action combined with throwing movements predisposes water polo players to musculoskeletal shoulder pain and injury $[10,11]$.

Water polo is a popular team sport played in South Africa both nationally, and internationally. South Africa has regularly competed in the FINA World Aquatic Champions in water polo. The South African male water polo team is currently ranked within the top 10 teams in the world [12]. Water polo is a popular aquatic sport that is incorporated into the secondary school sport programme in many South African schools [13]. Despite the national popularity and international success of this sport, there have been no studies published that documented the prevalence of musculoskeletal pain and/ or injury among South African water polo players. This lack of water polo sport epidemiological research leads players into a vicious cycle of practise/play, injury, rest, practise/play and re-injury. The identification of water polo related musculoskeletal pain and injury will help the coaches, exercise scientists and rehabilitative specialists to develop preventive strategic plans to enhance sport performance. The objective of this paper is to document the prevalence of water polo musculoskeletal pain among competitive adolescent male players residing in Kwa-Zulu Natal, South Africa.

\section{Material and methods}

All competitive male water polo players between the ages of 15-17 years who were members of the first team from the 10 premiere water polo schools in Kwa Zulu Natal were invited to voluntarily participate in the study $(n=110)$. From this pool of 10 schools only, nine participated via informed voluntary consent, thereby yielding a sample size of 100 . The tenth school refrained from participation due to time constraints of the school's busy sport and academic programmes. The sample secured represents $90.91 \%$ of the competitive male water polo players from the 10 premier water polo schools in Kwa Zulu Natal, South Africa. However there was $100 \%$ compliance among first team players of the nine schools that participated in the study. The statistical rule of thumb pertain to the minimum percentage of subjects participating in a study yielding power of significance of the findings of the study is $30 \%$ of the population being researched [14]. The Kwa-Zulu Natal high school provincial team is among the three strongest teams in South Africa [15]. The members from the Kwa-Zulu Natal team are predominantly selected from the schools who participated in the study.

Information on the participants' demographical, sport epidemiological and training history was obtained using a validated questionnaire (adapted from Van Heerden [16]). The fundamental problem concerning international sport epidemiological investigations is the inconsistent definition of musculoskeletal injury [17]. Hagglund et al. [17] proposed that the prevalence of musculoskeletal injury can be established, if the following has being documented; anatomical site of musculoskeletal pain, symptoms of musculoskeletal pain and intensity of pain that renders the person incapacitated or unable to play and/or practise sport. The definition of musculoskeletal pain employed in this study was any sensation of distress to the musculoskeletal system ranging from uncomfortable to worst pain ever experienced, which inhibited the water polo player from engaging in practise and/or competing for a minimum duration of 24 hours (adapted from van Herdeen [16]). Participants were requested to indicate only water polo-related musculoskeletal pain, and not musculoskeletal pain contracted from other sport and/or recreational activities. The combination of the subjects' identification of sensations of musculoskeletal pain (such as dull aching, discomfort, numbness, pins and needles, radiating), 
intensity of pain (accessed severity of pain according to the Kee and Seo Pain Rating Scale [18]) and anatomical location leads to the inference of musculoskeletal injury.

In addition posture profile analyses were conducted according to Kendall et al. [19] postural profile analyses protocol. This exploratory posture profile analyses was unique to water polo epidemiological investigations. Subjects stood against a posture profile chart in the anatomical position in both the frontal and sagittal planes. The plumb line represented the line of centre which divided the body equally into right and left when the subject stood in the frontal plane. The spinous process of the vertebrae was marked to indicate lateral deviation from the plumb line (line of centre) in the frontal plane, thereby identifying the presence of scoliosis [20]. Scoliosis is the lateral deviation of the vertebral column in frontal plane [20]. Similarly the line of centre divides the body anteriorly and posteriorly when the subject stood in the sagittal plane. The line of centre passes through the auditory meatus, acromion process, anterior sacro-iliac crest (ASIS), behind the patella and in front of the lateral malleolus (when analysing posture in the sagittal plane). Forward displacement of the acromion process and excessive flexion of the thoracic vertebrae in the sagittal plane indicates kyphosis. Kent [20] attributes the primary causative factor of kyphosis to be poor posture developed from an unequal pull of paraspinal muscles. Anterior deviation of the ASIS away from the line of centre in the sagittal plane indicates lordosis which places compressive stress on the posterior elements of the spine [20]. In addition subjects' body mass and stature were measured. The data were analysed descriptively and statistically using chi-square tests $(\mathrm{p} \leq$ $0.05)$.

\section{Results}

The cohort's demographical data such as age, body mass, stature and body mass index are found in table 1. The racial demographics of the cohort reveal 99 subjects were white and one black (African). The response rate was $100 \%$. Seventy-two percent of the subjects experienced water polo related musculoskeletal pain $(p<0.001)$. The anatomical sites most vulnerable to musculoskeletal pain were; shoulder $(51.04 \%)$, knee (23.95\%), vertebral column (17.71\%), upper limb (6.25\%), and lower limb (1.04\%) $(\mathrm{p}<0.0001)$ (figure 1). The type of musculoskeletal pain sensations experienced by the players were: sharp (57.31\%), dull ache (28.04\%), burning $(8.53 \%)$, radiating $(2.43 \%)$, pins and needles $(2.43 \%)$ and numbness $(1.21 \%),(\mathrm{p}<0.01)$. The intensity of musculoskeletal pain were; moderate $(48.27 \%)$, low (20.68\%), high $(20.68 \%)$, uncomfortable $(1.14 \%)$, and worst pain ever experienced $(9.19 \%)(p<0.01)$. The majority of the players attributed their musculoskeletal pain to over-training $(88.00 \%)(\mathrm{p}<0.001)$, followed by rapid rotational movements $(8.00 \%)$, collision with another player $(2.66 \%)$ and being struck with a ball $(1.33 \%)$. The players accumulatively trained and played water polo for a sum of 17357.86 minutes per year, yielding 96 occurrences of musculoskeletal pain over the retrospective 12 month period of analysis. The players trained and practised water polo for an average of 6.61 $( \pm 2.13)$ months within the last 12 months, averaging $5.05( \pm 2.46)$ sessions per week. Water polo players' warm-up included $2 \mathrm{~km}$ free style swim thereafter practise shooting at goal at an average of $39.01( \pm 16.26)$ shots per session. All water polo training sessions lasted 120 minutes.

The novel posture profile results indicate a total of 129 musculoskeletal abnormalities namely scoliosis, kyphosis and lordosis (table 2).

Tab. 1. Demographical data of the water polo players $(\mathrm{n}=100)$

\begin{tabular}{|l|c|}
\hline \multicolumn{1}{|c|}{ Variables } & Mean \\
\hline Age (years) & $16.34( \pm 0.98)$ \\
\hline Body mass $(\mathrm{kg})$ & $75.56( \pm 11.74)$ \\
\hline Stature $(\mathrm{m})$ & $1.79( \pm 0.06)$ \\
\hline Body mass index $\left(\mathrm{kg} / \mathrm{m}^{2}\right)$ & $23.46( \pm 3.02)$ \\
\hline
\end{tabular}

Tab. 2. The incidence of deviant musculoskeletal posture among the water polo players $(n=100)$

\begin{tabular}{|l|c|c|}
\hline \multicolumn{1}{|c|}{ Musculoskeletal condition } & $\begin{array}{c}\text { Number } \\
\text { of players }\end{array}$ & $\begin{array}{c}\text { Significance } \\
(\mathbf{p}<\mathbf{0 . 0 5})\end{array}$ \\
\hline Scoliosis & 63 & $\mathrm{p}<0.01$ \\
\hline Kyphosis & 36 & $\mathrm{p}<0.01$ \\
\hline Lordosis & 30 & $\mathrm{p}<0.01$ \\
\hline
\end{tabular}

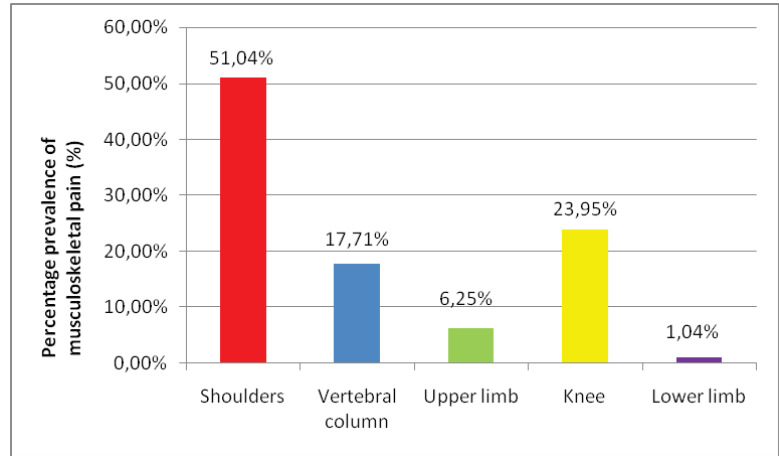

Fig. 1. Prevalence of water polo related musculoskeletal pain at various anatomical sites $(\mathrm{p}<0.0001)$

\section{Discussion}

Among the 100 water polo players surveyed, 72 experienced water polo-related musculoskeletal pain 
within the last 12 months $(\mathrm{p}<0.001)$. These findings correspond with other international injury surveys which recorded the prevalence of water polo-related musculoskeletal pain and/or injury ranging from $38 \%$ to $80 \%[7,9]$. Statistical interrogation of the data reveals that the shoulder was the anatomical site which sustained the most musculoskeletal pain followed by the vertebral column and knees $(p<0.0001)$. This finding concurs with previous reports that documented the prevalence of shoulder and knee musculoskeletal pain $[7,8,9]$.

The shoulder was identified as the most vulnerable anatomical site of musculoskeletal pain. Webster et al. [7] and Colville et al. [9] reported that the shoulder is a common anatomical site of musculoskeletal pain due to repetitive use in a water polo game. The musculature of the shoulder complex is frequently activated when player jostle to win possession of the ball, swim and throwing the ball at high velocity $[2,3]$. Normally water polo players abduct the humerus to $90^{\circ}$ and externally rotated their humerus (beyond $90^{\circ}$ ) to cock the arm and then explosively internally rotate the humerus and protract the scapular to generate high velocity when releasing the ball $[6,10]$. Shoulder abduction at $90^{\circ}$ normally is attained by the summation of $60^{\circ}$ glenohumeral abduction and $30^{\circ}$ scapular protraction [21]. Humeral external rotation is attained by concentric contraction of the teres minor and infraspinatus whilst internal rotation is accomplished by the concentric contraction of the subscapularis, pectorlais minor and major, latissimus dorsi and teres major [21]. The high prevalence of kyphotic posture among the water polo players who sustained shoulder musculoskeletal pain $(p<0.001)$ indicates taut pectoralis minor and subscapularis, which internally rotates the humerus. This internal humeral rotation alters the normal position of the greater tuberosity of the humerus to be under the lowest point of the acromion thereby decreasing the impingement interval between the humeral head and the acromion, causing the greater tuberosity to collide with the acromion (on humeral abduction), compressing the soft tissue thereby producing pain. The kyphotic posture also indicates an abnormal force couple relationship of the stronger internal shoulder rotators and the weaker external rotators.

In the deceleration phase (after the ball is released) external rotators decelerate the humerus in an attempt to prevent shoulder injury. Weak external rotators are unable to effectively decelerate the strong internal rotators resulting in excessive pain within the glenohumeral joint [3]. McMaster et al. [3] reported that the water polo players possessed stronger internal shoulder rotators and weaker external rotators, thereby establishing an abnormal force couple relationship [3]. This is illustrated by the kyphotic postures (taut pectoralis minor and subscapularis). Abnormal force couple relationships between internal and external glenohumeral rotators predispose athletes to glenohumeral pain and injury [22].

The knee was the anatomical site with second highest degree of musculoskeletal pain prevalence $(p<0.0001)$. Water polo players continually tread water using a breast stroke kick which produces a valgus stress on the medial aspect of the knee. Continuous breast stroke kicking in turbulent water increases the risk of medial collateral ligament sprains and muscle strains around the knee joint, producing symptoms of pain [22]. The game of water polo is very aggressive often producing violent kick aimed at knee and abdominal region [1]. Blows to the knee inhibit the recipient from treading water and thereby reduces his opportunity of gaining possession of the ball.

Musculoskeletal pain identified along the vertebral column, is a unique finding. There is no literature explaining the aetiology of the prevalence of water polo related vertebrae musculoskeletal pain and injury. The authors postulate that prevalence of vertebral musculoskeletal pain is attributed to the water polo players' kyphotic postures. Kyphosis is described as the forward displacement of the acromion process and excessive flexion of the thoracic vertebrae in the sagittal plane [20]. Taut pectoral minor, pectoral major, serratus anterior muscles pull the shoulder girdle and subsequently the thoracic vertebrae abnormally forward. This anterior thoracic vertebrae displacement produces spasms of the anterior muscles (indicated by dull aching pain), micro-tearing of the posterior muscles and intervertebral disc compression (indicated by pins and needle sensations) along the vertebral column. In addition kyphotic postures abnormally alter the force couple relationships between the anterior and posterior vertebral muscles, which lead to musculoskeletal pathology $[11,22]$. The kyphotic postures among water polo players are attributed to the high training mileage and the exclusive swim stroke selection. The cohort reported that their exclusive swim stroke of choice was the modified free-style. The biomechanical analysis of the modified free-style stroke is divided into the propulsion and recovery phases. In the propulsion phase, as the swimmer's hands penetrate the water, their shoulder undergoes adduction and internal rotation against the resistance of the water. During the recovery phase, shoulder undergoes abduction and external rotation against the resistance of the atmosphere (resistance of the water is greater than that of the atmosphere) $[3,5]$. Repetitive free-style swimming produces an abnormal force couple between the shoulder adductors (teres major, latissimus dorsi, pectoralis major) and abductors (supraspinatus, anterior and middle deltoid) in the frontal plane [21]. Similarly the shoulder internal rotators (teres major, pectoralis major, subscapularis, latissimus dorsi, and anterior deltoid) become stronger than shoulder 
external rotators (teres minor, infraspinatus and posterior deltoid) in the horizontal plane. These abnormal force couples pull the humeral head out of the glenoid fossa inferiorly and anteriorly, thereby stretching the superior and posterior shoulder musculature and ligament restraint, and thus manifesting the sensation of dull aching and sharp pain $[3,5]$.

The cohort was requested to subjectively describe the type of water polo-related musculoskeletal pain sensation they felt. The options to describe the pain were dull aching, sharp, discomfort, pins and needles, numbness and radiation. The type of pain sensation commonly experienced by those who sustained water polo-related musculoskeletal pain was dull aching, numbness, burning, sharp, radiating and pins and needles $(\mathrm{p}<0.01)$. Brukner and Khan [11] identify dull aching, sharp pain sensations as muscle pain. The sum of the dull aching and sharp sensations equal $85.35 \%$ indicative of muscle pathology. It is plausible that when players collide with each other when jostling for possession of the ball and/or being was struck with ball, the force of the blow penetrated the skin and was absorbed into the various muscle layers, thereby yielding symptoms of muscle pain such as dull aching and sharp sensations. The sum of the numbness, burning, radiating and pins and needles equal $14.65 \%$ suggestive of nerve pathology [22]. The combination of the most anatomical site of musculoskeletal pain, intensity of musculoskeletal pain assessed by the Kee and Seo pain rating scale [18] and type of musculoskeletal pain sensation is indicative of muscle and nerve pathology as suggested by Hagglund et al. [17].

\section{Conclusions}

The study concluded that the high school competitive male water polo players residing in Kwa-Zulu Natal, South Africa experienced a high prevalence of shoulder, knee and vertebral musculoskeletal pain. In addition to being the primary predisposing factor to the water polo related musculoskeletal pain, over training also produced deviant musculoskeletal posture.

\section{Acknowledgement}

The authors would like to extend their appreciation and gratitude to Mrs. N. Ellapen and Miss S. Abrahams for their assistance during the study.

\section{References}

1. Egan T. Water Polo: Rules, Tips, Strategy, and Safety. New York: The Rosen Publishing Group; 2004.

2. Elliot J. Shoulder pain and flexibility in elite water polo players. Physiotherapy 1993; 79(10): 693-7.
3. McMaster WC, Long SC, Caiozzo VJ. Isokinetic torque imbalances in the rotator cuff of elite water polo players. American Journal of Sports Medicine 1991; 19(1): 72-5.

4. Annett P, Fricker P, McDonald W. Injuries to elite male water polo players over a 13 year period. New Zealand Journal of Sport Medicine 2000; 28:78-83.

5. Giombini A, Rossi A, Pettrone FA, Dragoni S. Posterosuperior glenoid rim impingement as a cause of shoulder pain in top water polo players. Journal of Sport Medicine and Physical Fitness 1997; 37: 273-8.

6. Smith HK. Applied physiology of water polo. Sport Medicine 1998; 26(5): 317-34.

7. Webster MJ, Morris MN, Galna B. Shoulder pain in water polo: a systematic review of the literature. Australian Journal of Science and Medicine in Sport 2009; 12(1): 3-11.

8. Mountjoy M, Junge A, Alonso JM, Engebretsen L, Dragan I, Gerrard D, Kouidri M, Luebs E, Shahpar FM, Dvorak J. Sports injuries and illnesses in the 2009 FINA World Championships (Aquatics). British Journal of Sports Medicine 2010; 44(7): 522-7.

9. Colville JM, Markman BS. Competitive water polo. Clinical Sports Medicine 1999; 18(2): 305-11.

10. Witwer A, Sauers E. Clinical measures of shoulder mobility in college water polo players. Journal of Sports Rehabilitation 2006; 15: 45-57.

11. Brukner P, Khan K. Clinical Sport medicine. $3^{\text {rd }}$ ed. Sydney: McGraw-Hill; 2006.

12. Carnetti C. Water polo world ranking [serial online] 2011 Jan [cited 2011 Jan 31] URL:http:// www.fina.org/pool/index.php?option $=$ com wrapper\&view $=$ wrapper\&Itmed $=807$

13. Alswang J. South African Dictionary of Sport. South Africa: New Africa Books; 2003.

14. Terre-Blanche M, Durrheim K, Painter D. Research in Practice. Cape Town: University of Cape Town Press, 2008; p.50.

15. Morar B. High School South Africa male water polo team [serial online] 2011 Oct [cited Oct2011] URL:http://www.swimsa.org/Modules_BE/ AdminConsole/Contentmanager/data/file/SAMens-WCWaterPoloTeam-2011.pdf

16. Van Heerden HJ. Pre-participation evaluation and identification of aetiological risk factors in epidemiology of sports injuries among youths. Doctor of Philosophy. Pretoria: University of Pretoria; 1996.

17. Hagglund M, Walden M, Bahr R, Ekstrand J. Methods for epidemiological study of injuries to professional football players: developing the UEFA model. British Journal of Sport Medicine 2005; 39: 340- 346.

18. Kee D, Seo SR. Musculoskeletal disorders among nursing personnel in Korea. International Journal of Industrial Ergonomics 2007; 37: 207-212.

19. Kendall FP, McCreary EK, Provance PG, Rodgers MM, Romani WA. Muscles: Testing and Function with Posture and Pain ( $5^{\text {th }}$ edition). Lippincott Williams \& Wilkins, Philadelphia, USA; 2005.

20. Kent M. Oxford Dictionary of Sports Science and Medicine. United Kingdom: Oxford University Press; 2005.

21. Mansfield PJ, Neumann DA. Essentials of Kinesiology for the Physical Therapist Assistant. Mosby: Elsevier; 2008. 
21. Prentice WE. Rehabilitation Techniques for Sports Medicine and Athletic Training. Champaign, IL: Human Kinetics; 2004.
22. Bahr R, Maehlum S. Clinical Guide to Sport Injuries. Champaign, IL: Human Kinetics; 2004. 